\title{
Review Article \\ Intravenous Iron Administration and Hypophosphatemia in Clinical Practice
}

\author{
S. Hardy and X. Vandemergel \\ Department of General Internal Medicine, Centres Hospitaliers Jolimont, 1400 Nivelles, Belgium \\ Correspondence should be addressed to X. Vandemergel; xavier.vandemergel@jolimont.be
}

Received 29 January 2015; Revised 12 April 2015; Accepted 19 April 2015

Academic Editor: Bruce M. Rothschild

Copyright (C) 2015 S. Hardy and X. Vandemergel. This is an open access article distributed under the Creative Commons Attribution License, which permits unrestricted use, distribution, and reproduction in any medium, provided the original work is properly cited.

Introduction. Parenteral iron formulations are frequently used to correct iron deficiency anemia (IDA) and iron deficiency (ID). Intravenous formulation efficacy on ferritin and hemoglobin level improvement is greater than that of oral formulations while they are associated with lower gastrointestinal side effects. Ferric carboxymaltose- (FCM-) related hypophosphatemia is frequent and appears without clinical significance. The aim of this study was to assess the prevalence, duration, and potential consequences of hypophosphatemia after iron injection. Patients and Methods. The medical records of all patients who underwent parenteral iron injection between 2012 and 2014 were retrospectively reviewed. Pre- and postinjection hemoglobin, ferritin, plasma phosphate, creatinine, and vitamin D levels were assessed. Patients who developed moderate (range: $0.32-0.80 \mathrm{mmol} / \mathrm{L}$ ) or severe $(<0.32 \mathrm{mmol} / \mathrm{L})$ hypophosphatemia were questioned for symptoms. Results. During the study period, 234 patients received iron preparations but 104 were excluded because of missing data. Among the 130 patients included, 52 received iron sucrose (FS) and 78 FCM formulations. Among FS-treated patients, 22\% developed hypophosphatemia versus 51\% of FCM-treated patients, including $13 \%$ who developed profound hypophosphatemia. Hypophosphatemia severity correlated with the dose of FCM $(p=0.04)$ but not with the initial ferritin, hemoglobin, or vitamin D level. Mean hypophosphatemia duration was 6 months. No immediate clinical consequence was found except for persistent fatigue despite anemia correction in some patients. Conclusions. Hypophosphatemia is frequent after parenteral FCM injection and may have clinical consequences, including persistent fatigue. Further studies of chronic hypophosphatemia long-term consequences, especially bone assessments, are needed.

\section{Introduction}

In the United States, iron-deficiency anemia (IDA) affects 1$2 \%$ of adults while iron deficiency without anemia (ID) is more prevalent, affecting $11 \%$ of childbearing age women, and it is estimated that 7.8 million women have iron deficiency [1]. Symptoms of IDA usually include weakness, headache, irritability, fatigue, exercise intolerance and reduced exercise capacity [2], pagophagia, and restless legs syndrome [3]. They are often not specific and no clear clinical correlation has been shown between the degree of anemia and clinical manifestations. However, it has previously been shown that iron supplementation should be discussed in women with unexplained fatigue whose ferritin levels are less than $50 \mu \mathrm{g} / \mathrm{L}$, even in the absence of anemia [4]. Parenteral iron treatments are increasingly used because of their higher efficacy and fewer gastrointestinal side effects compared to oral iron therapy [5]. In Europe, ferric carboxymaltose (FCM) and iron sucrose (FS) are frequently used in this context.

Phosphate homeostasis is maintained via the bonekidney endocrine axis, which is mainly regulated by the parathyroid hormone (PTH), vitamin $\mathrm{D}$, and a group of hormones called phosphatonins, including fibroblast growth factor 23 (FGF23) [6]. In 2008 [7], the FDA has reported that $2.1 \%$ of patients treated with FCM injection developed hypophosphatemia but profound hypophosphatemia was rare. However, we have recently reported the case of a patient with profound and sustained hypophosphatemia due to phosphate diabetes [8] after FCM administration, a complication which has also been reported by others [9-11], and some patients, including those with history of bariatric surgery, are more likely to develop hypophosphatemia. Phosphate plays an important role in the organism and hypophosphatemia, especially when severe, is associated with various 
complications including fatigue [12], seizures [13], osmotic demyelination syndrome [14], myocardial depression [15], ventricular tachycardia [16], proximal myopathy [17], rhabdomyolysis [18], and hemolytic anemia [19]. The aim of this study was to retrospectively assess the impact of intravenous iron formulations on the phosphate level in clinical practice.

\section{Patients and Methods}

The medical records of all patients treated with parenteral iron formulation (either FCM or FS) in the Department of General Internal Medicine, Centres Hospitaliers Jolimont, Belgium (800 beds), between January 2012 and December 2014 were retrospectively reviewed. Indication, type, and dose of iron formulations were studied. Biological parameters including hemoglobin, ferritin, plasma phosphate, creatinine, $\mathrm{PTH}$, and vitamin D levels were assessed before and after iron injection. Hypophosphatemia was considered moderate when the phosphate level ranged between 0.32 and $0.80 \mathrm{mmol} / \mathrm{L}$ and severe when the level was less than $0.32 \mathrm{mmol} / \mathrm{L}$.

2.1. Statistical Analysis. Results are expressed as mean \pm standard deviation. Comparisons were done using a $t$-test. $p<0.05$ was considered statistically significant. Correlations were assessed using a Spearman test.

\section{Results}

During the study period, 234 patients received iron formulations. One hundred and four cases were excluded due to the absence of phosphate assessment before or after iron injection. Finally, 130 patients were included: 52 were treated with FS injection (17 men, 35 women) and 78 with FCM injection (24 men, 54 women).

3.1. Iron Sucrose. Among the 52 FS-treated patients, the mean dose of FS was $701 \mathrm{mg}$ (quartile 200-800). Patient mean age was $55 \pm 21$ years. Iron was prescribed by hematologists ( 5 patients), geriatricians [2], gastroenterologists [4], nephrologists [1], oncologists [4], internists [3], pediatricians [2], and gynecologists [1]. Indications for FS injection were IDA in 40 cases and ID in 6 cases and 3 patients had no anemia and no ID. Moreover, 3 patients received FS injection while their hemoglobin level and iron status were not assessed. Patient characteristics are presented in Table 1. Forty patients were asymptomatic, 12 presented with fatigue, and 3 complained of dyspnea. One patient had anemia-related vertigo. The mean hemoglobin level before injection was $10.1 \pm 1.99 \mathrm{gr} / \mathrm{dL}$ and it increased to $11.5 \pm 2.3 \mathrm{gr} / \mathrm{dL}$ after iron administration $(p=$ 0.02 ). The mean ferritin level rose from $64 \mu \mathrm{g} / \mathrm{L}$ (quartile: $6-$ $61 \mu \mathrm{g} / \mathrm{L})$ to $133 \mu \mathrm{g} / \mathrm{L}(34-139 \mu \mathrm{g} / \mathrm{L})(p=0.08)$. No difference in ferritin level or in hemoglobin level was noted between patients with and without fatigue ( $p=0.63$ and $p=0.6$ for the ferritin and hemoglobin level, resp.). Before intravenous injection, $42 \%$ of patients had taken oral iron formulation which was ineffective and led to digestive intolerance in 54\% and $31 \%$ of cases, respectively. The initial phosphate level
TABLE 1: Patient characteristics.

\begin{tabular}{lcc}
\hline & Iron sucrose & $\begin{array}{c}\text { Iron } \\
\text { carboxymaltose }\end{array}$ \\
\hline$N$ & 52 & 78 \\
Men/women & $17 / 35$ & $24 / 54$ \\
Active cancer & 7 & 16 \\
Chronic kidney disease & 7 & 10 \\
History of bariatric surgery & 5 & 15 \\
Inflammatory bowel disease & 5 & 4 \\
Gynecologic losses & 3 & 7 \\
Anemia & 40 & 70 \\
Iron deficiency without anemia & 6 & 3 \\
Fatigue & $23 \%$ & $29 \%$ \\
Asymptomatic & $76 \%$ & $67 \%$ \\
Phosphate level $<0.81$ mmol/L & $22 \%$ & $51 \%$ \\
Phosphate level $<0.32$ mmol/L & 0 & $13 \%$ \\
\hline
\end{tabular}

measured was $1.08 \pm 0.23 \mathrm{mmol} / \mathrm{L}$ and it did not change significantly following FS administration $(1.00 \pm 0.29 \mathrm{mmol} / \mathrm{L}$; $p=0.37)$. Before injection, 4 patients had moderate hypophosphatemia and none had severe hypophosphatemia. After injection, $22 \%$ of patients developed hypophosphatemia with a phosphate level less than $0.80 \mathrm{mmol} / \mathrm{L}$ (they were all within normal range before injection). The lowest value after administration was $0.44 \mathrm{mmol} / \mathrm{L}$. Hypophosphatemia duration varied between 2 and 18 weeks. However, the longterm phosphate level was not systematically assessed. No clinical manifestations of hypophosphatemia were found in the medical records. After injection, the phosphate level did not correlate with the initial hemoglobin level $(\mathrm{rs}=0.153$, $p=0.5)$, ferritin level $(\mathrm{rs}=0.05, p=0.82)$, or cumulated injected iron doses $(\mathrm{rs}=0.2, p=0.37)$.

3.2. Iron Carboxymaltose. Among the 78 FCM-treated patients, the mean dose of FCM was $2123 \mathrm{mg}$ (quartile: 1000$2000 \mathrm{mg}$ ). Various physicians prescribed the drug, including haematologists (6 cases), gastroenterologists [6], oncologists [2], general internists [2], nephrologists [1], pneumologists [1], endocrinologists [1], geriatricians [1], surgeons [1], and emergency physicians [1]. Ninety percent of patients had IDA, $4 \%$ had ID, and $6 \%$ had history of IDA but did not have anemia or ID at the time of the injection. Before injection, $52 \%$ of patients had taken oral iron which was associated with gastrointestinal side effects in $20 \%$ of cases.

The initial hemoglobin level was $9.6 \pm 1.6 \mathrm{gr} / \mathrm{dL}$ and it increased to $11.5 \pm 1.7 \mathrm{gr} / \mathrm{dL}$ after injection $(p<0.001)$. The ferritin level was also significantly higher after injection (from $63 \mu \mathrm{g} / \mathrm{L}(8-55)$ to $408 \mu \mathrm{g} / \mathrm{L}(128-536)$ ). No difference in hemoglobin level was observed between patients with and without symptoms of fatigue $(9.6 \pm 1.8 \mathrm{gr} / \mathrm{dL}$ versus $9.7 \pm$ $1.7 \mathrm{gr} / \mathrm{dL}$, resp.).

The initial mean phosphate level was $1.08 \pm 0.18 \mathrm{mmol} / \mathrm{L}$ and it decreased to $0.82 \pm 0.29 \mathrm{mmol} / \mathrm{L}$ following iron administration $(p<0.0001)$. After injection, $13 \%$ of patients had a phosphate level $<0.32 \mathrm{mmol} / \mathrm{L}$ and $51 \%$ had a phosphate level 
TABLE 2: Comparison between patients developing hypophosphatemia after FCM injection and patients without hypophosphatemia.

\begin{tabular}{lccc}
\hline & Patients with hypophosphatemia & Patients without hypophosphatemia & \\
\hline$N$ & 38 & 40 & \\
Women/men & $27 / 11$ & $27 / 13$ & NS \\
Age & $57 \pm 11$ & $58 \pm 19$ & $\mathrm{NS}$ \\
Vitamin D level before injection $(\mathrm{ng} / \mathrm{mL})$ & $21.9 \pm 16.9$ & $20 \pm 10$ & $\mathrm{NS}$ \\
Hemoglobin $(\mathrm{g} / \mathrm{L})$ & $9.5 \pm 1.3$ & $9.7 \pm 1.8$ & $\mathrm{NS}$ \\
Ferritin $(\mu \mathrm{g} / \mathrm{L})$ & $44(6.25-55)$ & $80(9-95)$ & $p=0.14$ \\
Cumulated dose $(\mathrm{mg})$ & $2092 \pm 2002$ & $1350 \pm 860$ & $p=0.04$ \\
\hline
\end{tabular}

$<0.80 \mathrm{mmol} / \mathrm{L}$. No difference in hypophosphatemia severity was noted between patients with history of bariatric surgery and the other patients.

Patients with severe hypophosphatemia were questioned about their symptoms, including $62 \%$ by phone interview. Among them, 55\% had fatigue improvement and 30\% complained of fatigue worsening while the remaining patients had no change in fatigue. In the group with fatigue worsening, the mean phosphate level was $0.55 \mathrm{mmol} / \mathrm{L}(0.32-0.76 \mathrm{mmol} / \mathrm{L})$. The mean hypophosphatemia duration was 6 months (2-9 months), a long period of time which was due to the fact that patients often received other injections during the follow-up. Some patients never reached a normal phosphate level during the entire study period ( 2 years). The comparison of patients with and without hypophosphatemia is presented in Table 2.

\section{Discussion}

Iron is an essential nutrient for the optimal functioning of the human body. It plays a central role in hemoglobin synthesis and in many cellular processes, including oxygen transport and storage, generation of energy through oxidative phosphorylation, and enzyme activity affecting the intracellular metabolism [20]. IDA is commonly found in clinical practice, affecting 1-2\% of adults in the United States [1] and accounting worldwide for 65.5 million years lived with disability [21]. Classical symptoms of IDA reported in the literature include fatigue, headache, dyspnea, and sleep disturbance secondary to restless legs syndrome [2, 22]. IDA is also known to negatively impact the physical quality of life and cognitive functions $[23,24]$.

However, the link between anemia and symptoms, for example, fatigue, has been poorly investigated in the literature. First, it is well known that patients may have acute or chronic profound anemia in the absence of symptoms $[25,26]$. The study by Wood and Elwood did not find a correlation between the hemoglobin level and the symptoms of anemia, including fatigue, dyspnea, and palpitations [27]. Most of the trials assessing the efficacy of intravenous iron formulations have shown a rapid increase in ferritin and hemoglobin levels, assessed as primary endpoints. Some of them have also assessed clinical endpoints. A sponsored, randomized, placebo-controlled, single-blind study [28] has assessed the efficacy and safety of single-dose intravenous FCM on fatigue reduction in iron-deficient, premenopausal women with symptomatic, unexplained fatigue and normal or borderline hemoglobin level ( $\geq 115 \mathrm{~g} / \mathrm{L})$. They have shown that a single infusion of FCM improved the fatigue, mental quality of life, cognitive function, and erythropoiesis in these patients. In their study, $28 \%$ of related adverse events were observed in the treatment group versus $3.4 \%$ in the placebo group ( $p<0.0004$ ), including fever, urticaria, headache, and nausea. Herfs et al. [29] have shown an improvement in some clinical symptoms with FCM, including fatigue, lack of concentration, hair loss, dyspnea, and sleep disturbance in women with IDA and ID. Froessler et al. [30] have also shown an improved quality of life related to FCM injections in pregnant women with anemia but they did not assess fatigue with a validated scale and they reported $20 \%$ of side effects. In patients with inflammatory bowel disease, Evstatiev et al. [31] have shown that the quality of life remained unchanged after FCM administration in nonanemic patients with low serum ferritin level. In heavy uterine bleeding, van Wyck et al. [32] have compared FCM to oral ferrous sulfate in 325 women with postpartum IDA with hemoglobin levels less than $10 \mathrm{gr} / \mathrm{L}$. They have shown that both treatment groups presented similar improvements in terms of quality of life scores. Another study by the same group [33] has also shown an improved quality of life in acute anemic patients. Finally, improved quality of life, functional capacity, or six-minute walk test has been reported in patients with chronic heart failure $[34,35]$.

In terms of efficacy, our study was similar to prospective studies showing a rapid elevation in hemoglobin and ferritin levels $[36,37]$. Regarding side effects, in clinical trials, serious anaphylactic/anaphylactoid reactions have been reported in $0.1 \%$ of patients treated with FCM [38]. Other serious reactions potentially related to hypersensitivity, including pruritus, rash, urticaria, wheezing, or hypotension, were experienced by $1.5 \%$ of cases versus $3.8 \%$ of patients with hypertension [39]. Hypophosphatemia is not systematically assessed in clinical trials but when done its rate is about $2.1 \%$ [38], while other studies have reported higher rates. In the study assessing the effect of a single dose of FCM in fatigued women, Favrat et al. [28] have indeed found that $86 \%$ of patients had a phosphate level $<0.8 \mathrm{mmol} / \mathrm{L}$ at day 7 which resolved spontaneously before the end of the study in almost all FCM-treated patients; however, no data on the risk of profound hypophosphatemia $(<0.32 \mathrm{mmol} / \mathrm{L})$, fatigue persistence, and hypophosphatemia development were reported. Iron-induced hypophosphatemia could play a role given that the results of some cognitive function tests do not improve 
after treatment. In patients with chronic kidney disease (CKD) and IDA, Macdougall et al. [40] have also found that the mean serum phosphate level decreased by $0.18 \mathrm{mmol} / \mathrm{L}$ at week 4 from baseline value but returned to normal value at week 52.

In 2010, Mani et al. [10] has reported a case of profound hypophosphatemia after FCM injection in a stable renal transplant recipient. Some case reports have been published since then [9-11] and we have recently published the case of a patient with severe hypophosphatemia due to phosphate diabetes secondary to FCM injection [8]. In our study, 22\% of patients developed hypophosphatemia after FS treatment but none of them developed severe hypophosphatemia. This frequency is high but hypophosphatemia was always moderate. We found only two case reports of hypophosphatemia after iron sucrose injection $[41,42]$.

Phosphate plays a key role in various biological processes. In recent years, new insights into the regulation of the phosphate metabolism have been obtained, including growing evidence suggesting that factors other than the $\mathrm{PTH}$ and vitamin $\mathrm{D}$ are involved in maintaining the phosphate balance. A new class of phosphate-regulating factors, the socalled "phosphatonins," has also been shown to play a role in phosphate-wasting diseases [43]. Among them, FGF23 is involved in various diseases, including autosomal dominant hypophosphatemic rickets/osteomalacia (ADHR) or tumorinduced osteomalacia [44]. True hypophosphatemia can be induced by a decrease in intestinal absorption, increase in urinary phosphate excretion, or acute movement of extracellular phosphate into the cells. Then, the normal renal response to phosphate depletion is to increase phosphate reabsorption, leading to the virtual abolition of urinary phosphate excretion. Most of the filtered phosphate is reabsorbed in the proximal tubule by the sodium-phosphate cotransporter in the luminal membrane [45]. A phosphate plasma level $<1 \mathrm{mg} / \mathrm{dL}(0.32 \mathrm{mmol} / \mathrm{L})$ can be deleterious because it can lead to cardiac arrest and severe hypophosphatemia can lead to metabolic encephalopathy and could therefore contribute to the development of central and extrapontine myelinolysis $[13,14,16]$.

In this study, $13 \%$ of patients developed severe and prolonged hypophosphatemia after FCM injection. We did not find any risk factor for the development of severe hypophosphatemia and we showed that a vitamin $\mathrm{D}$ level initially low did not correlate with the phosphate level following injection. The mechanism underlying the development of hypophosphatemia is not known but many arguments suggest a role of FGF23. Per se, the FGF23 level is increased in ID $[46,47]$ but, in our retrospective study, no correlation was found between the initial ferritin level and the postinjection hypophosphatemia severity. In a prospective trial, Schouten et al. have shown that intravenous iron polymaltose injection is followed by a rapid increase in FGF23 level and a drop in phosphate and $1,25(\mathrm{OH}) 2 \mathrm{D}$ tubular reabsorption that persisted 3 weeks after injection. The increased FGF23 level found in their study was very high and similar to that seen in ADHR [48]. We found no difference between patients developing hypophosphatemia and nonhypophosphatemic patients except for the iron cumulative dose. To the best of our knowledge, there are no published studies conducted in patients without CKD in which the impact of FCM injection on phosphate metabolism has been assessed. However, in a post hoc study, Prats et al. [49] have assessed the effect of FCM injection on the serum phosphate and c-terminal FGF23 levels in nondialysis CKD patients and no difference between hypophosphatemic and nonhypophosphatemic patients was found in terms of initial $25(\mathrm{OH}) \mathrm{D}$, ferritin, and hemoglobin levels.

Our study has some limitations, in particular its retrospective design and the fact that the value of the FGF23 level was not recorded. However, if hypophosphatemia is secondary to the increase in FGF23 as demonstrated in other studies, hypophosphatemia duration, several months in some cases, raises questions about the long-term side effects of the drug on bone.

Indeed, recent reports suggest that FGF23 may have a phosphate-independent effect on bones [50] with suppression of the osteoblast differentiation and matrix mineralization in a fetal rat calvaria cell line overexpressing FGF23. These consequences have been validated by two case reports of osteomalacia induced by intravenous iron sucrose administration [51, 52]. It is surprising because iron sucrose is less often complicated by hypophosphatemia. Therefore, patients with history of bariatric surgery need particular attention. They represented $18 \%$ of patients treated with FCM in our study. It is well known that metabolic bone disease is a concern during the follow-up of these patients [53] and an evolving concept of bariatric osteomalacia has emerged recently [54]. In our study, a history of bariatric surgery was not a risk factor for developing hypophosphatemia, but $60 \%$ of patients with history of bariatric surgery developed hypophosphatemia which, over the long term, could contribute to bone-specific complications. Moreover, $30 \%$ of our patients with FCM-induced hypophosphatemia complained about fatigue worsening. It is not known, however, if correcting hypophosphatemia could improve their symptomatology. At least, practitioners should keep in mind that it is indicated for ID. Indeed, in our study $3.8 \%$ of patients received iron formulations without hemoglobin or ferritin level evaluation. Blood test should be performed before injection and the phosphate level assessed both before and after injections.

Parenteral iron formulations are increasingly prescribed but their long-term side effects remain unknown, in particular the long-term bone impact of hypophosphatemia (13\% in our study). In cystic fibrosis patients, respiratory deterioration has been reported after treatment [55] and intravenous iron seems to have deleterious effects on mononuclear cells, oxidative stress, and apoptosis [56].

In conclusion, we found that parenteral FCM injection is often followed by hypophosphatemia which may be profound and last a long time without immediate clinical consequences except fatigue in some cases. Studies evaluating oral phosphate supplementation and its long-term consequences are needed because the biological change in phosphate homeostasis after injection is similar to that observed in diseases such as tumor-induced osteomalacia [57] or Xlinked hypophosphatemic rickets [58]. 


\section{Conflict of Interests}

The authors declare that there is no conflict of interests regarding the publication of this paper.

\section{References}

[1] A. C. Looker, P. R. Dallman, M. D. Carroll, E. W. Gunter, and C. L. Johnson, "Prevalence of iron deficiency in the United States," The Journal of the American Medical Association, vol. 277, no. 12, pp. 973-976, 1997.

[2] C. Cote, M. D. Zilberberg, S. H. Mody, L. J. Dordelly, and B. Celli, "Haemoglobin level and its clinical impact in a cohort of patients with COPD," European Respiratory Journal, vol. 29, no. 5, pp. 923-929, 2007.

[3] B. J. Bryant, Y. Y. Yau, S. M. Arceo, J. A. Hopkins, and S. F. Leitman, "Ascertainment of iron deficiency and depletion in blood donors through screening questions for pica and restless legs syndrome," Transfusion, vol. 53, no. 8, pp. 1637-1644, 2013.

[4] P. Vaucher, P.-L. Druais, S. Waldvogel, and B. Favrat, "Effect of iron supplementation on fatigue in nonanemic menstruating women with low ferritin: a randomized controlled trial," Canadian Medical Association Journal, vol. 184, no. 11, pp. 1247-1254, 2012.

[5] S. Lindgren, O. Wikman, R. Befrits et al., "Intravenous iron sucrose is superior to oral iron sulphate for correcting anaemia and restoring iron stores in IBD patients: a randomized, controlled, evaluator-blind, multicentre study," Scandinavian Journal of Gastroenterology, vol. 44, no. 7, pp. 838-845, 2009.

[6] C. Bergwitz and H. Jüppner, "Regulation of phosphate homeostasis by PTH, vitamin D, and FGF23," Annual Review of Medicine, vol. 61, pp. 91-104, 2010.

[7] http://www.accessdata.fda.gov/drugsatfda_docs/label /2013/203565s000lbl.pdf.

[8] X. Vandemergel and F. Vandergheynst, "Potentially lifethreatening phosphate diabetes induced by ferric carboxymaltose injection: a case report and review of the literature," Case Reports in Endocrinology, vol. 2014, Article ID 843689, 5 pages, 2014.

[9] A. Blazevic, J. M. Hunze, and J. M. M. Boots, "Severe hyposphosphatemia after intravenous iron administration," Netherlands Journal of Medicine, vol. 72, no. 1, pp. 49-53, 2014.

[10] L.-Y. Mani, G. Nseir, J.-P. Venetz, and M. Pascual, "Severe hypophosphatemia after intravenous administration of iron carboxymaltose in a stable renal transplant recipient," Transplantation, vol. 90, no. 7, pp. 804-805, 2010.

[11] Y. C. Fierz, R. Kenmeni, A. Gonthier, F. Lier, F. Pralong, and P. Coti Bertrand, "Severe and prolonged hypophosphatemia after intravenous iron administration in a malnourished patient," European Journal of Clinical Nutrition, vol. 68, no. 4, pp. 531533, 2014.

[12] P.-Y. Liu and C.-Y. Jeng, "Severe hypophosphatemia in a patient with diabetic ketoacidosis and acute respiratory failure," Journal of the Chinese Medical Association, vol. 67, no. 7, pp. 355-359, 2004.

[13] S. B. De Oliveira Iglesias, H. Pons Leite, and W. B. De Carvalho, "Hypophosphatemia-induced seizure in a child with diabetic ketoacidosis," Pediatric Emergency Care, vol. 25, no. 12, pp. 859861, 2009.
[14] J. Turnbull, D. Lumsden, A. Siddiqui, J.-P. Lin, and M. Lim, "Osmotic demyelination syndrome associated with hypophosphataemia: 2 cases and a review of literature," Acta Paediatrica, vol. 102, no. 4, pp. e164-e168, 2013.

[15] L. R. O'Connor, W. S. Wheeler, and J. E. Bethune, "Effect of hypophosphatemia on myocardial performance in man," The New England Journal of Medicine, vol. 297, no. 17, pp. 901-903, 1977.

[16] A. Ognibene, R. Ciniglio, A. Greifenstein et al., "Ventricular tachycardia in acute myocardial infarction: the role of hypophosphatemia," Southern Medical Journal, vol. 87, no. 1, pp. 65-69, 1994.

[17] J. A. Russell, “Osteomalacic myopathy," Muscle and Nerve, vol. 17, no. 6, pp. 578-580, 1994.

[18] P. C. Singhal, A. Kumar, L. Desroches, N. Gibbons, and J. Mattana, "Prevalence and predictors of rhabdomyolysis in patients with hypophosphatemia," American Journal of Medicine, vol. 92, no. 5, pp. 458-464, 1992.

[19] R. Poesen, D. Dierickx, and P. Evenepoel, "Hemolytic anemia associated with severe hypophosphatemia in a renal transplant recipient," Transplant International, vol. 25, no. 2, pp. e27-e28, 2012.

[20] J. L. Beard, "Iron biology in immune function, muscle metabolism and neuronal functioning," Journal of Nutrition, vol. 131, no. 2, pp. 568-580, 2001.

[21] N. J. Kassebaum, R. Jasrasaria, M. Naghavi et al., "A systematic analysis of global anemia burden from 1990 to 2010," Blood, vol. 123, no. 5, pp. 615-624, 2014.

[22] R. P. Allen, S. Auerbach, H. Bahrain, M. Auerbach, and C. J. Earley, "The prevalence and impact of restless legs syndrome on patients with iron deficiency anemia," The American Journal of Hematology, vol. 88, no. 4, pp. 261-264, 2013.

[23] C. W. Wells, S. Lewis, J. R. Barton, and S. Corbett, "Effects of changes in hemoglobin level on quality of life and cognitive function in inflammatory bowel disease patients," Inflammatory Bowel Diseases, vol. 12, no. 2, pp. 123-130, 2006.

[24] L. E. Murray-Kolb, "Iron status and neuropsychological consequences in women of reproductive age: what do we know and where are we headed?" Journal of Nutrition, vol. 141, no. 4, pp. 747s-755s, 2011.

[25] Q. Zhou, G. Lu, A. Liu, and T. Kohno, "Extraskeletal myxoid chondrosarcoma in the lung: asymptomatic lung mass with severe anemia," Diagnostic Pathology, vol. 7, article 112, 2012.

[26] J. M. Leung, R. B. Weiskopf, J. Feiner et al., "Electrocardiographic ST-segment changes during acute, severe isovolemic hemodilution in humans," Anesthesiology, vol. 93, no. 4, pp. 1004-1010, 2000.

[27] M. M. Wood and P. C. Elwood, "Symptoms of iron deficiency anaemia. A community survey," British Journal of Preventive \& Social Medicine, vol. 20, no. 3, pp. 117-121, 1966.

[28] B. Favrat, K. Balck, C. Breymann et al., "Evaluation of a single dose of ferric carboxymaltose in fatigued, iron-deficient women-PREFER a randomized, placebo-controlled study," PLoS ONE, vol. 9, no. 4, Article ID e94217, 2014.

[29] R. Herfs, L. Fleitmann, and I. Kocsis, "Treatment of iron deficiency with or without anaemia with intravenous ferric carboxymaltose in gynaecological practices-a non-interventional study," Geburtshilfe und Frauenheilkunde, vol. 74, no. 1, pp. 8188, 2014.

[30] B. Froessler, J. Collingwood, N. A. Hodyl, and G. Dekker, "Intravenous ferric carboxymaltose for anaemia in pregnancy," BMC Pregnancy and Childbirth, vol. 14, article 115, 2014. 
[31] R. Evstatiev, O. Alexeeva, B. Bokemeyer et al., "Ferric carboxymaltose prevents recurrence of anemia in patients with inflammatory bowel disease," Clinical Gastroenterology and Hepatology, vol. 11, no. 3, pp. 269-277, 2013.

[32] D. B. van Wyck, M. G. Martens, M. H. Seid, J. B. Baker, and A. Mangione, "Intravenous ferric carboxymaltose compared with oral iron in the treatment of postpartum anemia: a randomized controlled trial," Obstetrics and Gynecology, vol. 110, no. 2, pp. 267-278, 2007.

[33] L. T. Goodnough, D. B. Van Wyck, A. Mangione, J. Morrison, P. E. Hadley, and J. A. Jehle, "Large-dose intravenous ferric carboxymaltose injection for iron deficiency anemia in heavy uterine bleeding: a randomized, controlled trial," Transfusion, vol. 49, no. 12, pp. 2719-2728, 2009.

[34] S. D. Anker, J. C. Colet, G. Filippatos et al., "Ferric carboxymaltose in patients with heart failure and iron deficiency," The New England Journal of Medicine, vol. 361, no. 25, pp. 2436-2448, 2009.

[35] P. Ponikowski, D. J. van Veldhuisen, J. Comin-Colet et al., "Beneficial effects of long-term intravenous iron therapy with ferric carboxymaltose in patients with symptomatic heart failure and iron deficiency," European Heart Journal, vol. 36, no. 11, pp. 657668, 2015.

[36] W. Y. Qunibi, C. Martinez, M. Smith, J. Benjamin, A. Mangione, and S. D. Roger, "A randomized controlled trial comparing intravenous ferric carboxymaltose with oral iron for treatment of iron deficiency anaemia of non-dialysis-dependent chronic kidney disease patients," Nephrology Dialysis Transplantation, vol. 26, no. 5, pp. 1599-1607, 2011.

[37] C. Charytan, M. V. Bernardo, T. A. Koch, A. Butcher, D. Morris, and D. B. Bregman, "Intravenous ferric carboxymaltose versus standard medical care in the treatment of iron deficiency anemia in patients with chronic kidney disease: a randomized, active-controlled, multi-center study," Nephrology Dialysis Transplantation, vol. 28, no. 4, pp. 953-964, 2013.

[38] D. J. Cada, T. L. Levien, and D. E. Baker, "Ferric carboxymaltose," Hospital Pharmacy, vol. 49, no. 1, pp. 52-69, 2014.

[39] Injectafer, American Regent, Shirley, NJ, USA, 2013.

[40] I. Macdougall, A. H. Bock, F. Carrera et al., "FIND-CKD: a randomized trial of intravenous ferric carboxymaltose versus oral iron in patients with chronic kidney disease and iron deficiency anemia," Nephrology Dialysis Transplantation, vol. 29, no. 11, pp. 2075-2084, 2014.

[41] O. Konijki, S. Fukaya, H. Kanou, T. Imamoura, T. Iwamoto, and M. Takasaki, "A case of hypophosphatemia induced by intravenous administration of saccharated ion oxide," Nihon Ronen Igakkai Zasshi, vol. 10, pp. 805-810, 1994.

[42] Y. Shimizu, Y. Tada, M. Yamauchi et al., "Hypophosphatemia induced by intravenous administration of saccharated ferric oxide. Another form of FGF23-related hypophosphatemia," Bone, vol. 45, no. 4, pp. 814-816, 2009.

[43] T. Berndt and R. Kumar, "Phosphatonins and the regulation of phosphate homeostasis," Annual Review of Physiology, vol. 69, pp. 341-359, 2007.

[44] T. Saito and S. Fukumoto, "Fibroblast Growth Factor 23 (FGF23) and Disorders of Phosphate Metabolism," International Journal of Pediatric Endocrinology, vol. 2009, Article ID 496514, 2009.

[45] H. S. Murer, "Cellular mechanisms in proximal tubular Pi reabsorption: some answers and more questions," Journal of the American Society of Nephrology, vol. 2, no. 12, pp. 1649-1665, 1992.
[46] M. Wolf, T. A. Koch, and D. B. Bregman, "Effects of iron deficiency anemia and its treatment on fibroblast growth factor 23 and phosphate homeostasis in women," Journal of Bone and Mineral Research, vol. 28, no. 8, pp. 1793-1803, 2013.

[47] B. H. Durham, F. Joseph, L. M. Bailey, and W. D. Fraser, "The association of circulating ferritin with serum concentrations of fibroblast growth factor-23 measured by three commercial assays," Annals of Clinical Biochemistry, vol. 44, no. 5, pp. 463466, 2007.

[48] B. J. Schouten, P. J. Hunt, J. H. Livesey, C. M. Frampton, and S. G. Soule, "FGF23 elevation and hypophosphatemia after intravenous iron polymaltose: a prospective study," Journal of Clinical Endocrinology and Metabolism, vol. 94, no. 7, pp. 23322337, 2009.

[49] M. Prats, R. Font, C. García, C. Cabré, M. Jariod, and A. M. Vea, "Effect of ferric carboxymaltose on serum phosphate and C-terminal FGF23 levels in non-dialysis chronic kidney disease patients: post-hoc analysis of a prospective study," BMC Nephrology, vol. 14, article 167, 2013.

[50] H. Wang, Y. Yoshiko, R. Yamamoto et al., "Overexpression of fibroblast growth factor 23 suppresses osteoblast differentiation and matrix mineralization in vitro," Journal of Bone and Mineral Research, vol. 23, no. 6, pp. 939-948, 2008.

[51] K. Sato and M. Shiraki, "Saccharated ferric oxide-induced osteomalacia in Japan: iron-induced osteopathy due to nephropathy," Endocrine Journal, vol. 45, no. 4, pp. 431-439, 1998.

[52] S. Yamamoto, Y. Okada, H. Mori, S. Fukumoto, and Y. Tanaka, "Fibroblast growth factor 23-related osteomalacia caused by the prolonged administration of saccharated ferric oxide," Internal Medicine, vol. 51, no. 17, pp. 2375-2378, 2012.

[53] S. E. Williams, "Metabolic bone disease in the bariatric surgery patient," Journal of Obesity, vol. 2011, Article ID 634614, 9 pages, 2011.

[54] S. E. Williams and A. A. Licata, "Improved forearm bone mineral density after treatment with vitamin D3 in a patient 30 years after bariatric surgery," Journal of Bone and Mineral Research, vol. 23, article s226, 2008.

[55] Z. H. Hoo and M. J. Wildman, "Intravenous iron among cystic fibrosis patients," Journal of Cystic Fibrosis, vol. 11, no. 6, pp. 560562, 2012.

[56] A. Martin-Malo, A. Merino, J. Carracedo et al., "Effects of intravenous iron on mononuclear cells during the haemodialysis session," Nephrology Dialysis Transplantation, vol. 27, no. 6, pp. 2465-2471, 2012.

[57] R. Kumar, "Tumor-induced osteomalacia and the regulation of phosphate homeostasis," Bone, vol. 27, no. 3, pp. 333-338, 2000.

[58] G. I. Baroncelli, B. Toschi, and S. Bertelloni, "Hypophosphatemic rickets," Current Opinion in Endocrinology, Diabetes and Obesity, vol. 19, no. 6, pp. 460-467, 2012. 


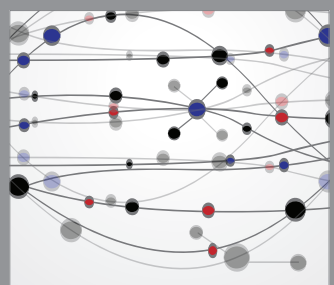

The Scientific World Journal
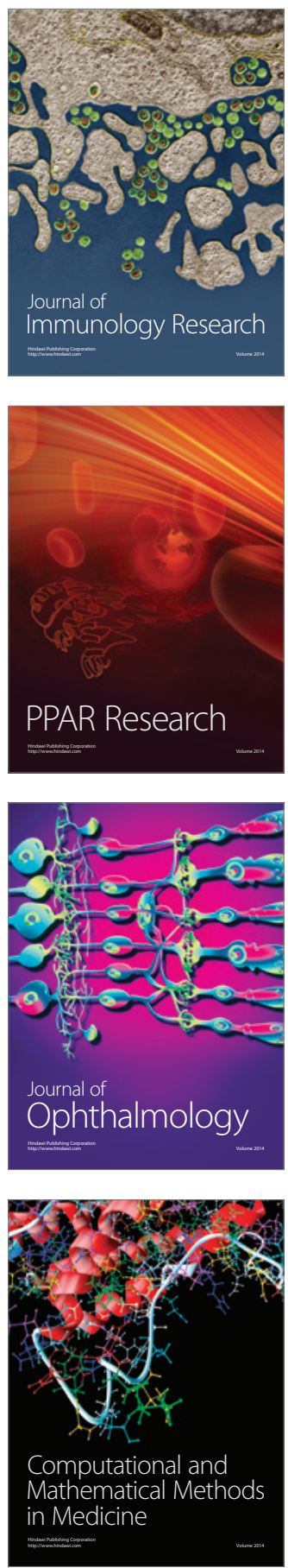

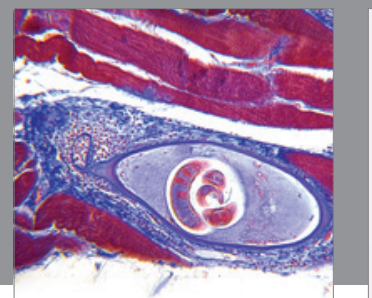

Gastroenterology

Research and Practice
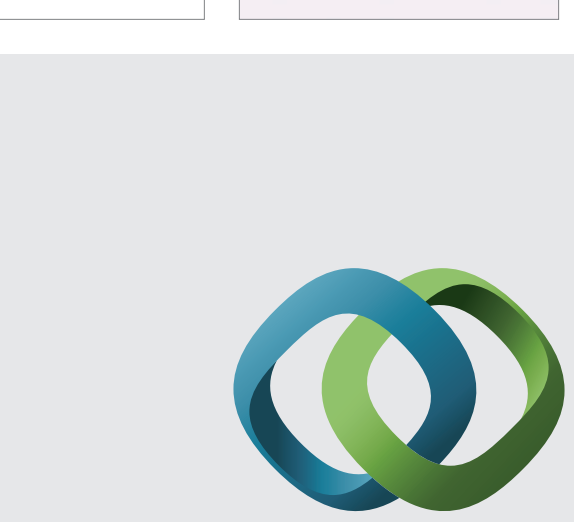

\section{Hindawi}

Submit your manuscripts at

http://www.hindawi.com
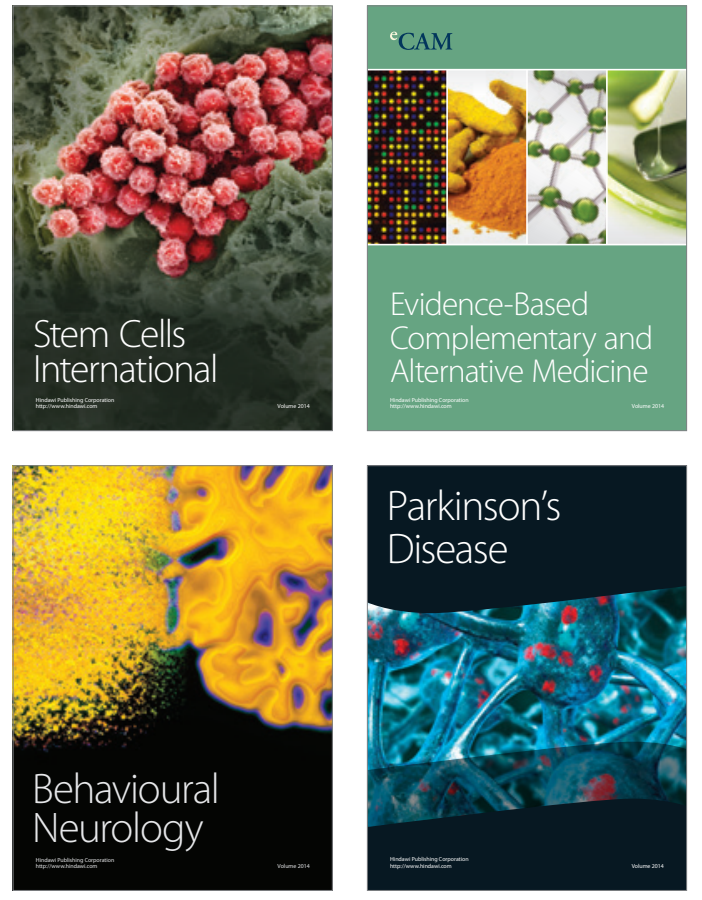
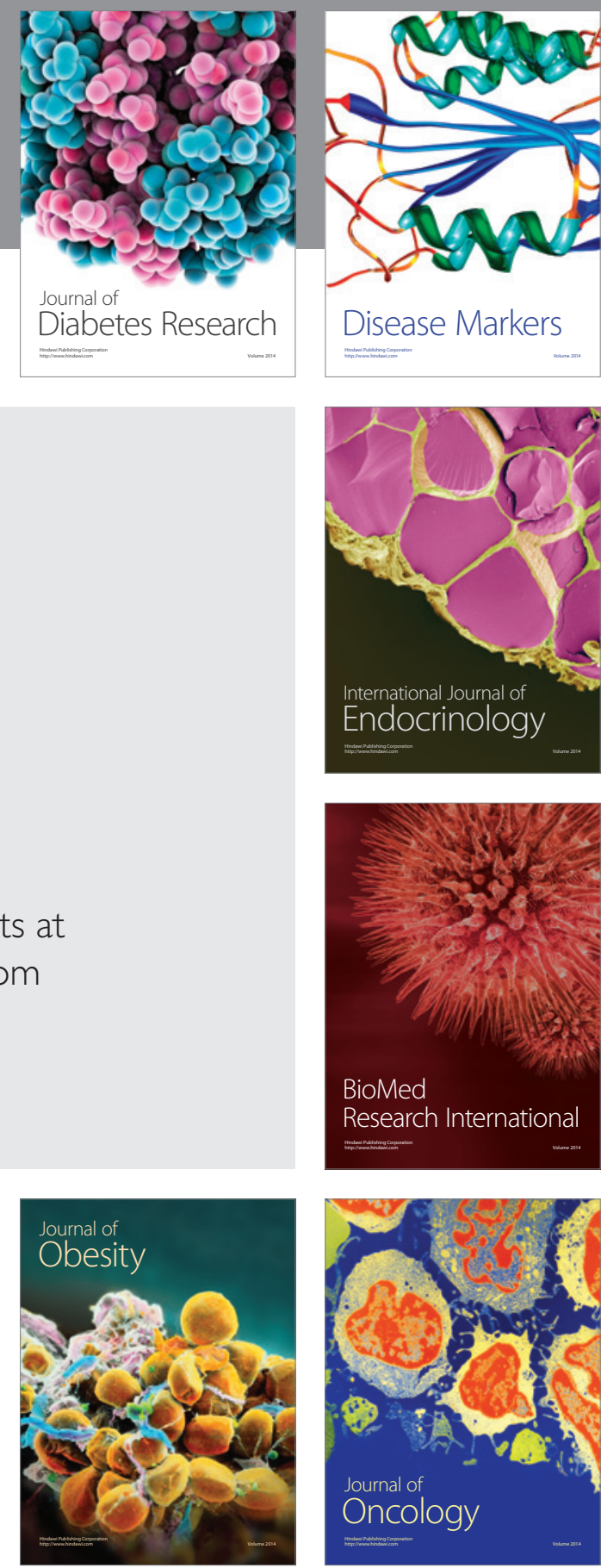

Disease Markers
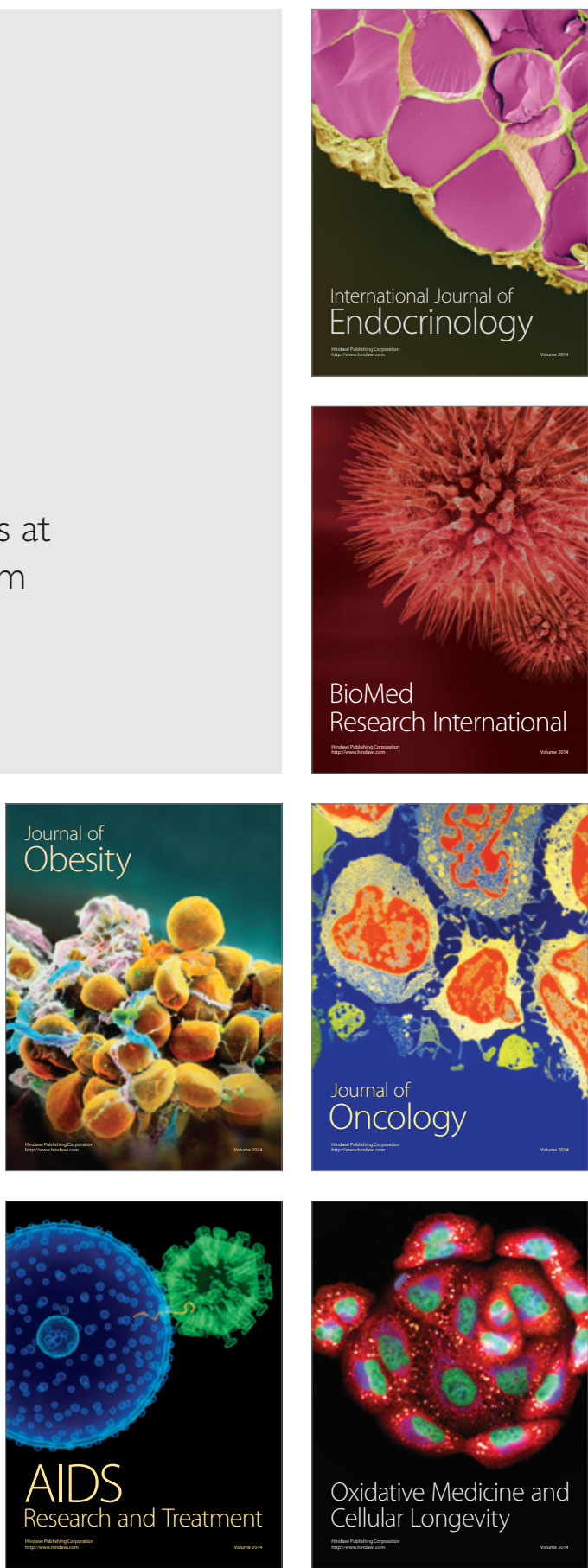